\title{
Convective Hydromagentic Slip Flow with Variable Properties Due to a Porous Rotating Disk
}

\author{
Mohammad M. Rahman
}

Department of Mathematics and Statistics, College of Science, Sultan Qaboos University, P.O. Box 36, Postal code 123, Muscat, Sultanate of Oman, Email:mansurdu@yahoo.com.

النسيل الحطل الإنزلفي لمائعمتخير الخصائص ، ناتج عن دورلنقرص مسلي

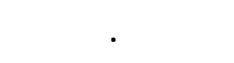

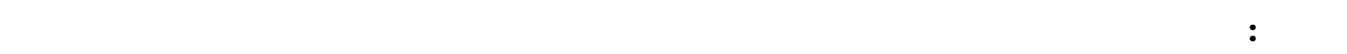

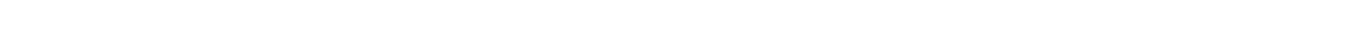

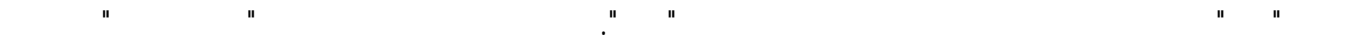

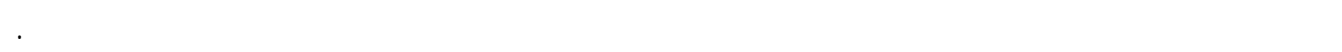

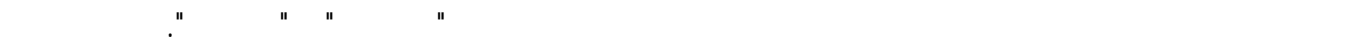

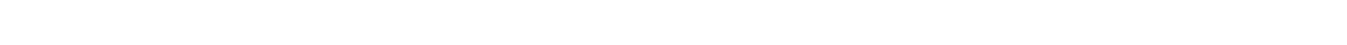

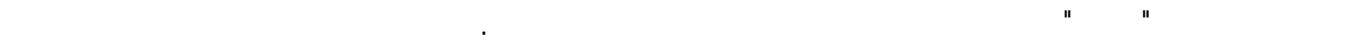
بشكل جوهري في خصائص الانزلاق و القل الحراري.

\begin{abstract}
In this paper we investigate convective heat transfer characteristics of steady hydromagnetic slip flow over a porous rotating disk taken into account the temperature dependent density, viscosity and thermal conductivity in the presence of Hall current, viscous dissipation and Joule heating. Using von-Karman similarity transformations we reduce the governing equations for flow and heat transfer into a system of ordinary differential equations which are highly nonlinear and coupled. The resulting nondimensional equations are solved numerically by applying Nachtsheim-Swigert iteration technique. The results show that when modeling a thermal boundary layer-with temperature dependent fluid properties, consideration of Prandtl number as constant within the boundary layer; produces unrealistic results. Therefore it must be treated as variable throughout the boundary layer. Results also show that the slip factor significantly controls the flow and heat transfer characteristics.
\end{abstract}

KEYWORDS: Rotating disk; Heat transfer; Convection; Slip flow; Variable properties. 


\section{Introduction}

Tn recent years, the flow dynamics due to a rotating disk, originating from the early formulation of von Karman (1921), has been a popular area of research. Since then many researchers (Cochran, 1934; Roger and Lance, 1960; Benton, 1965; Kuiken, 1971; Owen and Rogers, 1989; Herrero et al 1994; Kelson and Desseaux, 2000; Andrsson and Korte, 2002; Takhar et al 2002) have studied and reported results on disk-shaped bodies with or without heat transfer. Flow due to a rotating disk is encountered in many industrial, geothermal, geophysical, technological and engineering applications. A few of them are rotating heat exchangers, rotating disk reactors for bio-fuels production, computer disk drives, and gas or marine turbines.

\section{Nomenclature}

\begin{tabular}{|c|c|c|c|}
\hline$a$ & constant & $U_{t}$ & target velocity \\
\hline$b$ & constant & $u$ & velocity along radial direction \\
\hline B & magnetic field vector & $v$ & velocity along tangential direction \\
\hline$B_{0}$ & applied magnetic field & $w$ & velocity along axial direction \\
\hline$C f$ & skin friction coefficient & $w_{s}$ & non-dimensional suction velocity \\
\hline & specific heat at constant pressure & $w_{w}$ & suction velocity $x, y, z$ Cartesian \\
\hline $\mathrm{d}$ & constant & & coordinates \\
\hline $\mathbf{E}$ & electric field & Gre & Symbols \\
\hline$E c$ & Eckert number & $\beta$ & Hall factor \\
\hline$-e$ & charge of electron & $\gamma$ & relative temperature difference \\
\hline$F$ & dimensionless radial velocity & & parameter \\
\hline$G$ & dimensionless tangential velocity & $\rho$ & density of the fluid \\
\hline$H$ & dimensionless axial velocity & $\rho_{\infty}$ & density of the ambient fluid \\
\hline $\mathrm{Ha}$ & Hartman number & $\mu$ & coefficient of dynamic viscosity \\
\hline Kn & electric current density & $\mu_{\rho}$ & dynamic viscosity of the ambient \\
\hline$m$ & $\begin{array}{l}\text { Knuasen number } \\
\text { Hall current parameter }\end{array}$ & $\infty$ & fluid \\
\hline $\mathrm{Nu}$ & Nusselt number & $v_{\infty}$ & kinematic viscosity of the ambient \\
\hline$n_{e}$ & electron concentration per unit volume & & fluid \\
\hline$p$ & pressure within the boundary layer & $\sigma$ & $\begin{array}{l}\text { electric conductivity } \\
\text { thermal conductivity }\end{array}$ \\
\hline$p_{\infty}$ & pressure of the ambient fluid & $\kappa_{\infty}$ & thermal conductivity of the \\
\hline$p_{e}$ & electronic pressure & & ambient fluid \\
\hline Pr & variable Prandtl number & $\eta$ & similarity parameter \\
\hline $\mathrm{Pr}_{\infty}$ & ambient Prandtl number & $\xi$ & target momentum accommodation \\
\hline $\mathbf{q}$ & velocity vector & & coefficient \\
\hline$q_{w}$ & surface heat flux & $\lambda$ & mean free path \\
\hline $\mathrm{Re}$ & rotational Reynolds number & $\theta$ & dimensionless temperature \\
\hline$r$ & cylindrical radial coordinate & $\phi$ & tangential coordinate \\
\hline$T$ & temperature within boundary layer & $\Omega$ & angular velocity \\
\hline
\end{tabular}


The effects of an applied magnetic field on the steady flow due to the rotation of a disk of infinite or finite extent were studied by El-Mistikawy et al. (1991) and El-Mistikawy and Atia (1990). Atia and Aboul-Hassan (1997) studied steady hydromagnetic flow due to an infinite disk rotating with uniform angular velocity in the presence of an axial magnetic field. In their analysis they neglected the induced magnetic field but considered Hall current. Attia (1998) studied the effects of suction as well as injection in the presence of a magnetic field on the unsteady flow past a rotating porous disk. It was found that the combined effect of a magnetic field with strong injection may stabilize the growth of the boundary layer.

Sparrow et al. (1971) studied the flow of Newtonian fluid due to the rotation of a porous-surfaced disk with a set of linear slip-flow conditions. A substantial reduction in torque then occurred as a result of surface slip. Miklavcic and Wang (2004) further revisited the problem of Sparrow et al. and pointed out that the slipflow boundary conditions could also be used for slightly rarefied gases or for flow over grooved surfaces. Arikoglu and Ozkol (2006) studied MHD slip flow over a rotating disk with heat transfer. It is observed that both the slip factor and the magnetic flux decrease the velocity in all directions and thicken the thermal boundary layer. Recently, Osalusi et al. (2008) studied thermal-diffusion and diffusion-thermo effects on MHD slip flow due to a rotating disk.

In classical treatment of thermal boundary layers, fluid properties (such as density, viscosity, thermal conductivity) are assumed to be constant; however, experiments indicate that this assumption only makes sense if temperature does not change rapidly for the application of interest. To predict the flow behavior accurately, it may be necessary to take into account these variable properties. Zakerullah and Ackroyd (1979) investigated free convection flow above a horizontal circular disk considering variable fluid properties. In the case of fully developed laminar flow in concentric annuli, the effect of the variable property has been investigated by Herwig and Klemp (1988). Atia (2006) studied unsteady hydromagnetic flow due to an infinite rotating disk, considering temperature dependent viscosity in a porous medium with Hall and ion-slip currents. Maleque and Sattar (2005a) studied the effect of variable properties on the steady laminar convective flow due to a rotating disk while Maleque and Sattar (2005b) further investigated the same problem in the presence of Hall current. Osalusi and Sibanda (2006) revisited the problem of Maleque and Sattar (2005a), considering magnetic effect.

When fluid properties such as viscosity and thermal conductivity vary with temperature, Prandtl number (see section 2) varies too. All of these afore-mentioned works considered Prandtl number as constant within the boundary layer, although viscosity and thermal conductivity depends on temperature. Hence one of the motivations behind this study is also to investigate how variable Prandtl number affects the flow and heat transfer characteristics.

In the present study we extend the work of Maleque and Sattar (2005b) and analyze the flow and heat transfer characteristics in the presence of viscous dissipation and Joule heating, considering slip flow boundary condition at the surface of a uniformly heated rotating disk. The resulting governing equations are solved numerically applying Nachtsheim-Swigert (1965) iteration technique. Graphical results for non-dimensional velocity and temperature profiles including skin-friction coefficient and the Nusselt number in tabular form are presented for a range of values of the parameters characterizing the flow. The accompanying discussion provides physical interpretations of the results.

\section{Mathematical Model}

Let us consider a steady hydromagnetic laminar flow of an electrically conducting fluid due to a porous rotating disk of infinite extent in the presence of an external uniform magnetic field directed perpendicular to the disk. The fluid properties are taken as strong functions of temperature. A uniform suction or injection through the disk is considered for the whole range of suction or injection velocities. 


\subsection{Basic Equations}

The equations governing the steady hydromagnetic laminar convective flow are: Equation of continuity:

$$
\nabla \cdot(\rho \mathbf{q})=0
$$

Navier-Stokes equation:

$$
\rho(\mathbf{q} \cdot \nabla) \mathbf{q}=-\nabla p+[\nabla \cdot(\mu \nabla)] \mathbf{q}+(\mathbf{J} \times \mathbf{B}),
$$

Ohm's law for a moving conductor with Hall currents:

$$
\mathbf{J}=\sigma\left[\mathbf{E}+\mathbf{q} \times \mathbf{B}-\beta(\mathbf{J} \times \mathbf{B})+\beta \nabla p_{e}\right]
$$

Maxwell electromagnetic equations:

$$
\nabla . \mathbf{J}=0, \nabla \times \mathbf{E}=\mathbf{0}, \nabla . \mathbf{B}=0
$$

Energy equation:

$$
\begin{gathered}
\rho C_{p}(\mathbf{q} \cdot \nabla) T=\nabla \cdot(\kappa \nabla) T+\frac{J^{2}}{\sigma}+\mu \Phi \\
\Phi=2\left[\left(\frac{\partial u}{\partial x}\right)^{2}+\left(\frac{\partial v}{\partial y}\right)^{2}+\left(\frac{\partial w}{\partial z}\right)^{2}\right]+\left(\frac{\partial u}{\partial y}+\frac{\partial v}{\partial x}\right)^{2}+\left(\frac{\partial v}{\partial z}+\frac{\partial w}{\partial y}\right)^{2} \\
+\left(\frac{\partial w}{\partial x}+\frac{\partial u}{\partial z}\right)^{2}-\frac{2}{3}(\nabla \cdot \mathbf{q})^{2}
\end{gathered}
$$

Here $\mathbf{q}$ is the velocity vector, $\mathbf{B}$ is the magnetic field vector, $\mathbf{E}$ denotes the electrical field vector which results from charge separation and is in the $Z$-direction, $\mathbf{J}$ is the current density vector, $p$ is the pressure, $\rho$ is the density of the fluid, $\mu$ is the viscosity of the fluid, $\sigma$ is the electrical conductivity of the fluid, $\kappa$ is the thermal conductivity of the fluid, $C_{p}$ is the specific heat of the fluid, $T$ is the temperature of the fluid, and $\Phi$ is the viscous dissipation function. In equation (3) the term $\sigma \beta(\mathbf{J} \times \mathbf{B})$ denotes the Hall effects where $\beta=\frac{1}{e n_{e}}$ designates the Hall factor, $-e$ is the charge of electron, $n_{e}$ is the electron concentration per unit volume and $p_{e}$ is the electronic pressure. In equation (5) the term $\frac{J^{2}}{\sigma}$ represents Joule heating whereas $\mu \Phi$ is the viscous dissipation or frictional heating effects.

\subsection{Governing equations}

In non-rotating cylindrical polar coordinates $(r, \phi, z)$, let us consider a disk which rotates with constant angular velocity $\Omega$ about the $Z$-axis. The disk is placed at $Z=0$, and the fluid occupies the region $Z>0$, 


\section{CONVECTIVE HYDROMAGNETIC SLIP FLOW}

where $Z$ is the vertical axis in the cylindrical coordinates system with $r$ and $\phi$ as the radial and tangential axes respectively. The components of the flow velocity $\mathbf{q}$ are $(u, v, w)$ in the directions of increasing $(r, \phi, z)$ respectively. The surface of the rotating disk is maintained at a uniform temperature $T_{w}$ and far away from the wall, the free stream is kept at a constant temperature $T_{\infty}$ and at a constant pressure $p_{\infty}$. The fluid is assumed to be Newtonian, viscous and electrically conducting. An external uniform magnetic field is applied in the $Z$ direction. The electron-atom collision frequency is assumed to be relatively high so that the Hall effect cannot be neglected. Ion-slip effects are however ignored in the present analysis.

From equation (4), using the relation $\nabla \cdot \mathbf{B}=0$ for the magnetic field $\mathbf{B}=\left(B_{x}, B_{y}, B_{z}\right)$, we obtain that $B_{z}=B_{0}$ (constant) everywhere in the fluid. This assumption is valid only when the magnetic Reynolds number is very small so that magnetic induction effects can be ignored. For the current density $\mathbf{J}=\left(J_{x}, J_{y}, J_{z}\right)$ we obtain from the relation $\nabla \cdot \mathbf{J}=0$ that $J_{z}=$ constant . Hence we consider that the disk is non-conducting and therefore $J_{z}=0$ at the disk and hence zero everywhere. Finally we consider the case of a short circuit problem in which the applied electric field $\mathbf{E}=\mathbf{0}$ and also assume that the induced magnetic field is negligible in comparison with the applied magnetic field.

In the absence of electric field $\mathbf{E}$ and electron pressure $p_{e}$ equation (3) becomes

$$
\mathbf{J}=\left[\frac{\sigma B_{0}(v+m u)}{1+m^{2}}, \frac{\sigma B_{0}(m v-u)}{1+m^{2}}, 0\right] \text {, }
$$

where $m=\sigma \beta B_{0}$ is called Hall current parameter. It can be further shown that

$$
\mathbf{J} \times \mathbf{B}=\left[\frac{\sigma B_{0}^{2}(m v-u)}{1+m^{2}},-\frac{\sigma B_{0}^{2}(v+m u)}{1+m^{2}}, 0\right]
$$

We also assume that the fluid properties, viscosity $(\mu)$, thermal conductivity $(\kappa)$ and density $(\rho)$ are functions of temperature alone and obey the following laws (see Jayaraj, 1995; later used by Malek and Sattar, 1995b; Osalusi and Sibanda, 2006)

$$
\mu=\mu_{\infty}\left[T / T_{\infty}\right]^{a}, \kappa=\kappa_{\infty}\left[T / T_{\infty}\right]^{b}, \rho=\rho_{\infty}\left[T / T_{\infty}\right]^{d},
$$

where $a, b$ and $d$ are arbitrary exponents while $\mu_{\infty}, \kappa_{\infty}$ and $\rho_{\infty}$ are the viscosity, thermal conductivity and density of the ambient fluid respectively.

The flow configurations and geometrical coordinates are shown in Figure 1. Due to steady axially symmetric, compressible hydromagnetic laminar flow of a homogeneous fluid the governing equations take the following form (see Malek and Sattar, 1995b):

$$
\frac{\partial}{\partial r}(\rho r u)+\frac{\partial}{\partial z}(\rho r w)=0,
$$




$$
\begin{gathered}
\rho\left(u \frac{\partial u}{\partial r}-\frac{v^{2}}{r}+w \frac{\partial u}{\partial z}\right)=-\frac{\partial p}{\partial r}+\frac{\partial}{\partial r}\left(\mu \frac{\partial u}{\partial r}\right)+\frac{\partial}{\partial r}\left(\mu \frac{u}{r}\right)+\frac{\partial}{\partial z}\left(\mu \frac{\partial u}{\partial z}\right)+\frac{\sigma B_{0}^{2}}{\left(1+m^{2}\right)}(m v-u), \\
\rho\left(u \frac{\partial v}{\partial r}+\frac{u v}{r}+w \frac{\partial v}{\partial z}\right)=\frac{\partial}{\partial r}\left(\mu \frac{\partial v}{\partial r}\right)+\frac{\partial}{\partial r}\left(\mu \frac{v}{r}\right)+\frac{\partial}{\partial z}\left(\mu \frac{\partial v}{\partial z}\right)-\frac{\sigma B_{0}^{2}}{\left(1+m^{2}\right)}(v+m u),
\end{gathered}
$$

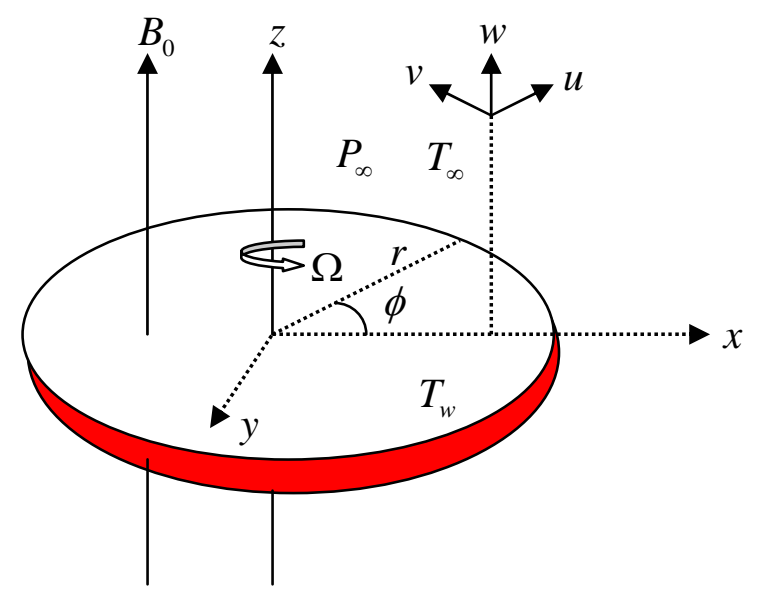

Figure 1. Flow configurations and coordinate system

$$
\begin{aligned}
\rho\left(u \frac{\partial w}{\partial r}+w \frac{\partial w}{\partial z}\right) & =-\frac{\partial p}{\partial z}+\frac{\partial}{\partial r}\left(\mu \frac{\partial w}{\partial r}\right)+\frac{1}{r} \frac{\partial}{\partial r}(\mu w)+\frac{\partial}{\partial z}\left(\mu \frac{\partial w}{\partial z}\right) \\
\rho C_{p}\left(u \frac{\partial T}{\partial r}+w \frac{\partial T}{\partial z}\right) & =\frac{\partial}{\partial r}\left(\kappa \frac{\partial T}{\partial r}\right)+\frac{\kappa}{r} \frac{\partial T}{\partial r}+\frac{\partial}{\partial z}\left(\kappa \frac{\partial T}{\partial z}\right)+\mu\left[\left(\frac{\partial u}{\partial z}\right)^{2}+\left(\frac{\partial v}{\partial z}\right)^{2}\right]+ \\
& \frac{\sigma B_{0}^{2}}{\left(1+m^{2}\right)}\left(u^{2}+v^{2}\right)
\end{aligned}
$$

If the mean free path of the fluid particles is comparable to the characteristic dimensions of the flow field domain, the assumption of continuum media is no longer valid, and as a consequence Navier-Stokes equation breaks down. In the range $0.1<K n<10$ of Knudsen number, the high order continuum equations (Burnett equations) should be used. For the range of $0.001<K n<0.1$, no-slip boundary conditions cannot be used and should be replaced with the following expression (Gad-el-Hak, 1999):

$$
U_{t}=\frac{2-\xi}{\xi} \lambda \frac{\partial u}{\partial z}
$$




\section{CONVECTIVE HYDROMAGNETIC SLIP FLOW}

where $U_{t}$ is the target velocity, $\xi$ is the target momentum accommodation coefficient and $\lambda$ is the mean free path. For $K n<0.001$, the no-slip boundary condition is valid; therefore, the velocity at the surface is equal to zero. In this study the slip and the no-slip regimes of the Knudsen number that lies in the range $0<K n<0.1$ are considered.

\subsection{Boundary conditions}

By using equation (15), the appropriate boundary conditions for our model are

(i) On the surface of the disk $(Z=0)$ :

$$
\begin{aligned}
u=U_{t}, v=\Omega r+U_{t}, w & =w_{w} \text { (slip flow and permeable surface conditions), } \\
T & =T_{w} \text { (uniform surface temperature). }
\end{aligned}
$$

(ii) Matching with the quiescent free stream $(z \rightarrow \infty)$ :

$$
u=0, v=0, T=T_{\infty}, p=p_{\infty} .
$$

\section{Transformation of the model}

To obtain the solutions of the governing equations (10)-(14) together with the boundary conditions (16) we introduce a dimensionless normal distance from the disk, $\eta=z\left(\Omega / v_{\infty}\right)^{1 / 2}$ along with the von-Karman transformation

$$
\left.\begin{array}{l}
u=\Omega r F(\eta), v=\Omega r G(\eta), w=\left(\Omega v_{\infty}\right)^{1 / 2} H(\eta), \\
p-p_{\infty}=2 \rho_{\infty} v_{\infty} \Omega P(\eta), T-T_{\infty}=\Delta T \theta(\eta),
\end{array}\right\}
$$

where $v_{\infty}$ is the kinematic viscosity of the ambient fluid and $\Delta T=T_{w}-T_{\infty}$.

Now substituting (17) into (10)-(14) we obtain the following nonlinear ordinary differential equations

$$
\begin{gathered}
H^{\prime}+2 F+\gamma d H \theta^{\prime}(1+\gamma \theta)^{-1}=0, \\
F^{\prime \prime}+a \gamma(1+\gamma \theta)^{-1} \theta^{\prime} F^{\prime}-\left[F^{2}-G^{2}+H F^{\prime}\right](1+\gamma \theta)^{d-a}+\frac{H a^{2}}{1+m^{2}}(m G-F)(1+\gamma \theta)^{-a}=0, \\
G^{\prime \prime}+a \gamma(1+\gamma \theta)^{-1} \theta^{\prime} G^{\prime}-\left[2 F G+H G^{\prime}\right](1+\gamma \theta)^{d-a}-\frac{H a^{2}}{1+m^{2}}(G+m F)(1+\gamma \theta)^{-a}=0, \\
\theta^{\prime \prime}+b \gamma(1+\gamma \theta)^{-1} \theta^{\prime 2}-\operatorname{Pr}_{\infty} H \theta^{\prime}(1+\gamma \theta)^{d-b}+\operatorname{Pr}_{\infty} E c \frac{H a^{2}}{1+m^{2}}(1+\gamma \theta)^{-b}\left(F^{2}+G^{2}\right)+ \\
\operatorname{Pr}_{\infty} E c(1+\gamma \theta)^{a-b}\left(F^{\prime 2}+G^{\prime 2}\right)=0,
\end{gathered}
$$

where $H a=B_{0}\left(\sigma / \rho_{\infty} \Omega\right)^{1 / 2}$ is the Hartmann number, $\operatorname{Pr}_{\infty}=\mu_{\infty} C_{p} / \kappa_{\infty}$ is the ambient Prandtl number, $E c=(\Omega r)^{2} / C_{p} \Delta T$ is the Eckert number and $\gamma=\Delta T / T_{\infty}$ is the relative temperature difference parameter, which is positive for a heated surface, negative for a cooled surface and zero for uniform properties. Thus by using (17) boundary conditions (16) become

$$
F=\varepsilon F^{\prime}, G=1+\varepsilon G^{\prime}, H=w_{s}, \theta=1 \text { at } \eta=0,
$$




$$
F=0, G=0, P=0, \theta=0 \text { as } \eta \rightarrow \infty \text {, }
$$

where $\varepsilon=\frac{2-\xi}{\xi} \lambda\left(\Omega / v_{\infty}\right)^{1 / 2}$ is the slip factor and $w_{s}=w\left(\Omega v_{\infty}\right)^{-1 / 2}$ represents a uniform suction when $w_{s}<0$ and uniform injection when $w_{s}>0$ at the surface of the disk.

\subsection{Particular cases}

A number of special cases can be derived from the full transformed momentum and energy equations (18)-(21) with the boundary conditions (22) which are as follows:

\begin{tabular}{|c|c|c|c|c|}
\hline i & $\gamma=w_{s}=0$ & without heat transfer & $\begin{array}{l}\text { no-slip } \\
\text { condition }\end{array}$ & Hassan and Attia (1997) \\
\hline ii & $\gamma=H a^{2}=m=E c=0$ & $\begin{array}{l}\text { heat transfer without } \\
\text { Joule heating }\end{array}$ & $\begin{array}{l}\text { no-slip } \\
\text { condition }\end{array}$ & $\begin{array}{l}\text { Kelson and Desseaux } \\
\text { (2000) }\end{array}$ \\
\hline iii & $\gamma=H a^{2}=m=0$ & without heat transfer & slip condition & $\begin{array}{l}\text { Miklavcic and Wang } \\
\text { (2004) }\end{array}$ \\
\hline iv & $\gamma=w_{s}=m=E c=0$ & $\begin{array}{l}\text { heat transfer without } \\
\text { Joule heating }\end{array}$ & slip condition & $\begin{array}{c}\text { Arikoglu and Ozkol } \\
\text { (2006) }\end{array}$ \\
\hline $\mathrm{V}$ & $H a^{2}=m=E c=0$ & $\begin{array}{l}\text { heat transfer without } \\
\text { Joule heating }\end{array}$ & $\begin{array}{l}\text { no-slip } \\
\text { condition }\end{array}$ & $\begin{array}{c}\text { Maleque and Sattar } \\
\text { (2005a) }\end{array}$ \\
\hline $\mathrm{vi}$ & $m=E c=0$ & $\begin{array}{l}\text { heat transfer without } \\
\text { Joule heating }\end{array}$ & $\begin{array}{c}\text { no-slip } \\
\text { condition }\end{array}$ & $\begin{array}{l}\text { Osalusi and Sibanda } \\
\text { (2006) }\end{array}$ \\
\hline vii & $E c=0$ & $\begin{array}{l}\text { heat transfer without } \\
\text { Joule heating }\end{array}$ & $\begin{array}{l}\text { no-slip } \\
\text { condition }\end{array}$ & $\begin{array}{l}\text { Maleque and Sattar } \\
\text { (2005b) }\end{array}$ \\
\hline
\end{tabular}

\section{Variable Prandtl Number}

The Prandtl number is a function of viscosity and as viscosity varies across the boundary layer, the Prandtl number varies, too. The assumption of constant Prandtl number inside the boundary layer may produce unrealistic results. Therefore, Prandtl number related to the variable viscosity is defined by

$$
\operatorname{Pr}=\frac{\mu C_{p}}{\kappa}=\frac{\mu_{\infty}(1+\gamma \theta)^{a} C_{p}}{\kappa_{\infty}(1+\gamma \theta)^{b}}=\frac{\mu_{\infty} C_{p}}{\kappa_{\infty}}(1+\gamma \theta)^{a-b}=\operatorname{Pr}_{\infty}(1+\gamma \theta)^{a-b}
$$

At the surface $(\eta=0)$ of the disk, this can be written as

$$
\operatorname{Pr}_{w}=\operatorname{Pr}_{\infty}(1+\gamma)^{a-b}
$$

From equation (23) it can be seen that for $\gamma \rightarrow 0$, the variable Prandtl number Pr equals the ambient Prandtl number $\operatorname{Pr}_{\infty}$. For $\eta \rightarrow \infty$ that is outside the boundary layer, $\theta(\eta)$ becomes zero. Therefore $\operatorname{Pr}$ equals $\operatorname{Pr}_{\infty}$ regardless of the values of $\gamma$.

Table 1 shows the variation of the Prandtl number at the surface of the disk for several values of $\gamma$ for a fixed value of the ambient Prandtl number $\operatorname{Pr}_{\infty}=0.64$ and the exponents $a=0.7, b=0.83$. From this table we see that for a positive value of $\gamma$, Prandtl number at the surface of the disk $\operatorname{Pr}_{w}$ decreases as $\gamma$ increases. The opposite effect is observed when $\gamma$ is negative. It must be noted that for $\gamma \leq-1$ no physically viable solutions exist. 


\section{CONVECTIVE HYDROMAGNETIC SLIP FLOW}

Table 1. Values of $\operatorname{Pr}$ versus $\gamma$ for $\operatorname{Pr}_{\infty}=0.64, a=0.7, b=0.83$ at $\eta=0$.

\begin{tabular}{|c|c|c|c|c|c|c|c|c|c|}
\hline$\gamma$ & -0.8 & -0.5 & -0.2 & 0.0 & 0.2 & 0.5 & 1.0 & 3.0 & 5.0 \\
\hline $\operatorname{Pr}$ & 0.789 & 0.700 & 0.659 & 0.640 & 0.625 & 0.607 & 0.585 & 0.534 & 0.507 \\
\hline
\end{tabular}

In light of the above discussions, using (23) the non-dimensional temperature equation (21) can be rewritten as

$$
\begin{aligned}
& \theta^{\prime \prime}+b \gamma(1+\gamma \theta)^{-1} \theta^{\prime 2}-\operatorname{Pr}(1+\gamma \theta)^{d-a} H \theta^{\prime}+ \\
& H a^{2} /\left(1+m^{2}\right) \operatorname{Pr} E c(1+\gamma \theta)^{-a}\left(F^{2}+G^{2}\right)+\operatorname{Pr} E c\left(F^{\prime 2}+G^{\prime 2}\right)=0 .
\end{aligned}
$$

Equation (25) is the corrected non-dimensional form of the energy equation in which Prandtl number is treated as variable. It is mentionable that this correction does not appear in the literature.

\section{Parameters of engineering interest}

The parameters of engineering interest for the present problem are the skin-friction coefficient $(C f)$ and the Nusselt number ( $\mathrm{Nu}$ ) which indicate physically wall shear stress and rate of heat transfer respectively. The action of the variable properties in the fluid adjacent to the disk sets up a tangential shear stress, which opposes the rotation of the disk. As a consequence, it is necessary to provide a torque at the shaft to maintain a steady rotation. The radial shear stress $\tau_{r}$ and tangential shear stress $\tau_{t}$ are defined by:

$$
\begin{aligned}
& \tau_{r}=\left[\mu\left(\frac{\partial u}{\partial z}+\frac{\partial w}{\partial r}\right)\right]_{z=0}=\mu_{\infty}(1+\gamma)^{a} \operatorname{Re}^{1 / 2} \Omega F^{\prime}(0), \\
& \tau_{t}=\left[\mu\left(\frac{\partial v}{\partial z}+\frac{1}{r} \frac{\partial w}{\partial \phi}\right)\right]_{z=0}=\mu_{\infty}(1+\gamma)^{a} \operatorname{Re}^{1 / 2} \Omega G^{\prime}(0) .
\end{aligned}
$$

Hence the skin-frictions ( $C f=\tau / \rho_{\infty} \Omega^{2} r^{2}$ ) along radial and tangential directions are obtained as

$$
\begin{aligned}
& C f_{r}=(1+\gamma)^{a} \operatorname{Re}^{-1 / 2} F^{\prime}(0), \\
& C f_{t}=(1+\gamma)^{a} \operatorname{Re}^{-1 / 2} G^{\prime}(0) .
\end{aligned}
$$

The rate of heat transfer from the disk surface to the fluid is computed by the application of Fourier's law as given below

$$
q_{w}=-\left(\kappa \frac{\partial T}{\partial z}\right)_{z=0}=-\kappa_{\infty} \Delta T(1+\gamma)^{b}\left(\frac{\Omega}{v_{\infty}}\right)^{1 / 2} \theta^{\prime}(0) .
$$

Hence the Nusselt number ( $\left.N u=\frac{r q_{w}}{\kappa_{\infty} \Delta T}\right)$ is obtained as

$$
N u=-(1+\gamma)^{b} \operatorname{Re}^{1 / 2} \theta^{\prime}(0),
$$

where $\operatorname{Re}=\Omega r^{2} / v_{\infty}$ is the rotational Reynolds number. Thus from equations (28), (29) and (31) we see that skin-friction coefficient and Nusselt number are proportional to the numerical values of $F^{\prime}(0), G^{\prime}(0)$ and $-\theta^{\prime}(0)$ which are calculated in the process of integration when solving the corresponding differential equations. 


\section{Method of solutions}

The set of equations (18)-(20) and (25) are highly nonlinear and coupled and therefore the system cannot be solved analytically. The system of transformed governing equations (18)-(20) and (25) with boundary conditions (12) is solved numerically using shooting method similar to that described by Nachtsheim-Swigert (1965). In equation (22) there are three asymptotic boundary conditions and hence three unknown surface conditions $F^{\prime}(0), G^{\prime}(0)$ and $\theta^{\prime}(0)$. Nachtsheim-Swigert developed an iteration technique to overcome the difficulties of determining the guess values of the unknown surface boundary conditions required for the shooting method. Within the context of the initial value method and the Nachtsheim-Swigert shooting iteration technique the outer boundary conditions may be functionally represented by

$$
\Psi_{j}\left(\eta_{\max }\right)=\Psi_{j}\left(F^{\prime}(0), G^{\prime}(0), \theta^{\prime}(0)\right)=\delta_{j}, j=1,2 \cdots 6,
$$

where $\Psi_{1}=F, \Psi_{2}=G, \Psi_{3}=\theta, \Psi_{4}=F^{\prime}, \Psi_{5}=G^{\prime}, \Psi_{6}=\theta^{\prime}$. The last three of these represents asymptotic convergence criteria.

Choosing $F^{\prime}(0)=g_{1}, G^{\prime}(0)=g_{2}$ and $\theta^{\prime}(0)=g_{3}$ and expanding in a first-order Taylor's series after using equations (26) yields

$$
\Psi_{j}\left(\eta_{\max }\right)=\Psi_{j, C}\left(\eta_{\max }\right)+\sum_{i=1}^{3} \frac{\partial \Psi_{j}}{\partial g_{i}} \Delta g_{i}=\delta_{j}, j=1,2 \cdots 6
$$

where subscript ' $C$ ' indicates the value of the function at $\eta_{\max }$ determined from the trial integration.

Solution of these equations in a least-square sense requires determining the minimum value of

$$
\Pi=\sum_{j=1}^{6} \delta_{j}^{2}
$$

with respect to $g_{i}(i=1,2,3)$.

Now differentiating $\prod$ with respect to $g_{i}$ we obtain

$$
\sum_{j=1}^{6} \delta_{j} \frac{\partial \delta_{j}}{\partial g_{i}}=0
$$

Substituting equation (27) into (29) after some algebra we obtain

where

$$
\sum_{k=1}^{3} a_{i k} \Delta g_{k}=b_{i}, i=1,2,3,
$$

Now solving the system of linear equations (30) we obtain the missing (unspecified) values of $g_{i}$ as

$$
g_{i} \cong g_{i}+\Delta g_{i}
$$

Thus adopting this numerical technique aforementioned, a computer program was set up for the solutions of the governing non-linear ordinary differential equations (18)-(20) and (25) of our problem where the integration technique was adopted as a sixth-order Runge-Kutta method of integration. The velocity and temperature are determined as a function of the coordinate $\eta$ and displayed graphically. 


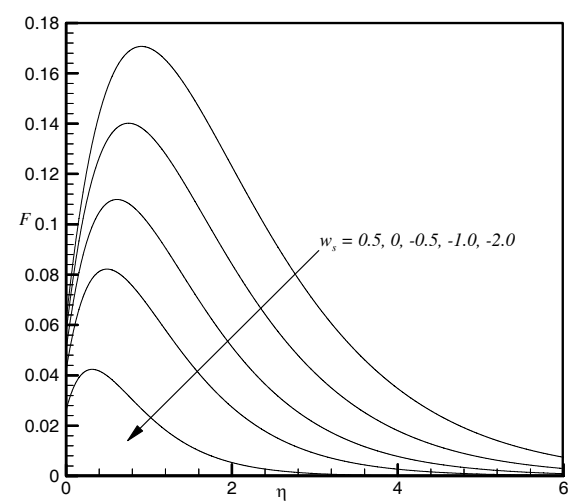

(a)

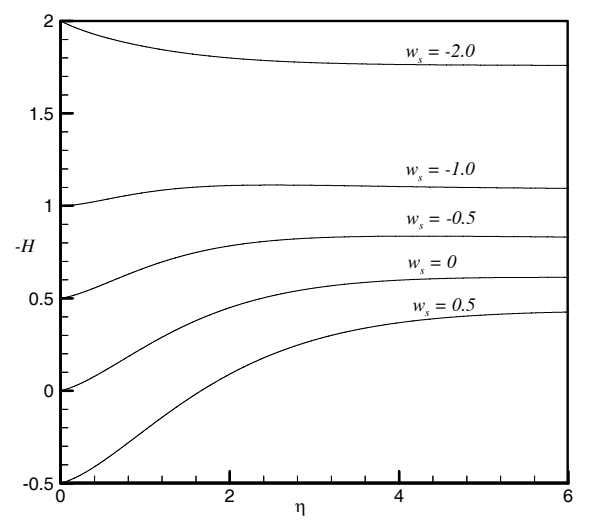

(c)

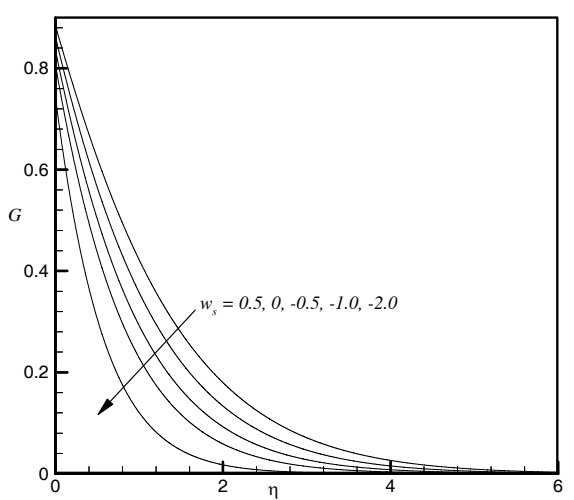

(b)

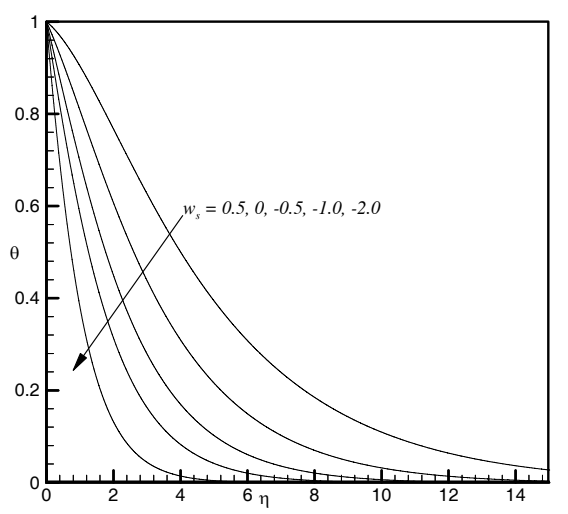

(d)

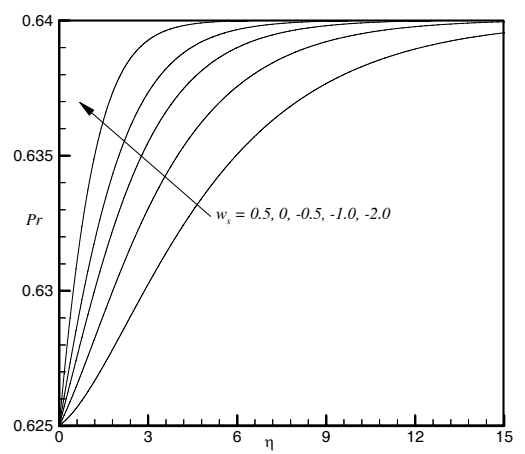

(e)

Figure 2. Variation of (a) radial velocity, (b) tangential velocity, (c) axial velocity, (d) temperature profile, and (e) variable Prandtl number for several values of $w_{s}$. 


\subsection{Numerical experiment}

In this paper, the effects of Hall current, viscous dissipation and Joule heating on a steady hydromagnetic convective slip flow of a viscous, Newtonian, electrically conducting fluid with variable properties over a rotating porous disk have been investigated numerically by using Nachtsheim-Swigert shooting iteration technique. It can be seen that the solutions are affected by the seven parameters, namely suction (or injection) parameter $w_{s}$, magnetic field parameter (or Hartmann number) $H a$, Hall current parameter $m$, relative temperature difference parameter $\gamma$, Prandtl number $\operatorname{Pr}$, Eckert number $E c$ and slip parameter $\varepsilon$. Since experimental data of the physical parameters are not available, in the numerical simulations the choice of the values of the parameters was dictated by the values chosen by the previous investigators.

For the present investigation we considered our working fluid as flue gas. For flue gases (ambient Prandtl number, $\operatorname{Pr}_{\infty}=0.64$ ) the values of the exponents $a, b$ and $d$ are taken as $a=0.7, b=0.83$ and $d=-1.0$ (see Jayaraj, 1995). The default values of the other parameters which we considered are $w_{s}=-1.0$, $H a^{2}=0.5, m=0.5, \gamma=0.2, \operatorname{Pr}=0.625, E c=0.2$, and $\varepsilon=0.2$ unless otherwise specified.

\subsection{Code verification}

To assess the accuracy of the present code, we reproduced the values of $F^{\prime}(0), G^{\prime}(0), H(\infty)$ and $\theta^{\prime}(0)$ for constant property models of Kelson and Desseaux (2000) (herein and after referred as KD2000) (see case-ii in section 3.1) and Arikoglu and Ozkol (2006) (herein and after referred as AO2006) (see case-iv in section 3.1). Tables 2-4 show the comparisons of the data produced by the present code and those of KD2000 and AO2006. In fact the results show a close agreement, and hence justify the use of the present code for the current model.

Table 2. Numerical values of $F^{\prime}(0),-G^{\prime}(0)$ and $-\theta^{\prime}(0)$ for various values of $w_{s}$ with $H a^{2}=m=E c=\gamma=0$ and $\operatorname{Pr}=0.71$.

\begin{tabular}{|c|r|r|r|r|r|r|}
\hline \multirow{2}{*}{$w_{s}$} & \multicolumn{2}{|c|}{$F^{\prime}(0)$} & \multicolumn{2}{c|}{$-G^{\prime}(0)$} & \multicolumn{2}{c|}{$-\theta^{\prime}(0)$} \\
\cline { 2 - 7 } & Present & KD2000 & Present & KD2000 & Present & KD2000 \\
\hline 4 & 0.24304404 & 0.243044 & 0.02892121 & 0.0289211 & 0.00001075 & 0.0000107 \\
\hline 3 & 0.30914768 & 0.309147 & 0.06028945 & 0.0602893 & 0.00057793 & 0.000576 \\
\hline 2 & 0.39893387 & 0.398934 & 0.13595275 & 0.135952 & 0.01103604 & 0.011013 \\
\hline 1 & 0.48948057 & 0.489481 & 0.30217432 & 0.302173 & 0.08504687 & 0.084884 \\
\hline 0 & 0.51022378 & 0.510233 & 0.61592380 & 0.615922 & 0.32637889 & 0.325856 \\
\hline-1 & 0.38954065 & 0.389569 & 1.17526180 & 1.175222 & 0.79393633 & 0.793048 \\
\hline-2 & 0.24241310 & 0.242421 & 2.03859590 & 2.038527 & 1.43876482 & 1.437782 \\
\hline-3 & 0.16558828 & 0.165582 & 3.0122231 & 3.012142 & 2.13677058 & 2.135585 \\
\hline-4 & 0.12475268 & 0.124742 & 4.00526266 & 4.005180 & 2.84369011 & 2.842381 \\
\hline
\end{tabular}




\section{CONVECTIVE HYDROMAGNETIC SLIP FLOW}

Table 3. Numerical values of $F^{\prime}(0)$ and $-G^{\prime}(0)$ for various values of $\varepsilon$ with $w_{s}=H a^{2}=m=E c=\gamma=0$ and $\operatorname{Pr}=0.71$.

\begin{tabular}{|c|c|c|c|c|}
\hline \multirow{2}{*}{$\varepsilon$} & \multicolumn{2}{|c|}{$F^{\prime}(0)$} & \multicolumn{2}{c|}{$-G^{\prime}(0)$} \\
\cline { 2 - 5 } & Present & AO2006 & Present & AO2006 \\
\hline 0.0 & 0.51022378 & 0.51023261 & 0.61592380 & 0.61592201 \\
\hline 0.1 & 0.42144560 & 0.42145363 & 0.60583699 & 0.60583524 \\
\hline 0.2 & 0.35257377 & 0.35258100 & 0.58367858 & 0.58367676 \\
\hline 0.5 & 0.22384294 & 0.22384820 & 0.50281179 & 0.50280970 \\
\hline 1.0 & 0.12792035 & 0.12792364 & 0.39492982 & 0.39492759 \\
\hline 2.0 & 0.06100834 & 0.06101009 & 0.27337241 & 0.27337013 \\
\hline 5.0 & 0.01858796 & 0.01858852 & 0.14339025 & 0.14338820 \\
\hline 10 & 0.00681240 & 0.00681255 & 0.08103175 & 0.08103008 \\
\hline 20 & 0.00236161 & 0.00236159 & 0.04378973 & 0.04378846 \\
\hline
\end{tabular}

Table 4. Numerical values of $-H(\infty)$ and $-\theta^{\prime}(0)$ for various values of $\varepsilon$ with $w_{s}=H a^{2}=m=E c=\gamma=0$ and $\operatorname{Pr}=0.71$.

\begin{tabular}{|c|c|c|c|c|}
\hline \multirow{2}{*}{$\varepsilon$} & \multicolumn{2}{|c|}{$-H(\infty)$} & \multicolumn{2}{c|}{$-\theta^{\prime}(0)$} \\
\cline { 2 - 5 } & Present & AO2006 & Present & AO2006 \\
\hline 0.0 & 0.88344324 & 0.8844741 & 0.32637889 & 0.32586063 \\
\hline 0.1 & 0.88055012 & 0.8813642 & 0.33402796 & 0.33349695 \\
\hline 0.2 & 0.87334256 & 0.8739572 & 0.33732324 & 0.33678090 \\
\hline 0.5 & 0.84230103 & 0.8423926 & 0.33521597 & 0.33465287 \\
\hline 1.0 & 0.79003973 & 0.7894772 & 0.32099888 & 0.32043299 \\
\hline 2.0 & 0.71185974 & 0.7103133 & 0.29357940 & 0.29299798 \\
\hline 5.0 & 0.58730981 & 0.5837646 & 0.24466400 & 0.24440461 \\
\hline 10 & 0.49317208 & 0.4875846 & 0.20570012 & 0.20504924 \\
\hline 20 & 0.40816322 & 0.3999758 & 0.16953552 & 0.16882963 \\
\hline
\end{tabular}

\subsection{Effect of step size}

To see the effects of the integration step size $\Delta \eta$, we ran the code for our model with three different step sizes namely $\Delta \eta=0.01, \Delta \eta=0.005$, and $\Delta \eta=0.001$. In each case, we found excellent agreement among the results. It was also found that $\Delta \eta=0.001$ provided sufficiently accurate (error less than $10^{-6}$ ) results and further refinement of the grid size was therefore not warranted.

\section{Results and discussion}

For the purpose of discussing the results, the numerical calculations are presented in the form of nondimensional velocity (radial, tangential and axial) and temperature profiles. In the calculations the values of the parameters namely suction (or injection) parameter $w_{s}$, magnetic field parameter (or Hartmann number) $\mathrm{Ha}$, Hall current parameter $m$, relative temperature difference parameter $\gamma$, Prandtl number Pr, Eckert number Ec and slip parameter $\varepsilon$ are varied. 


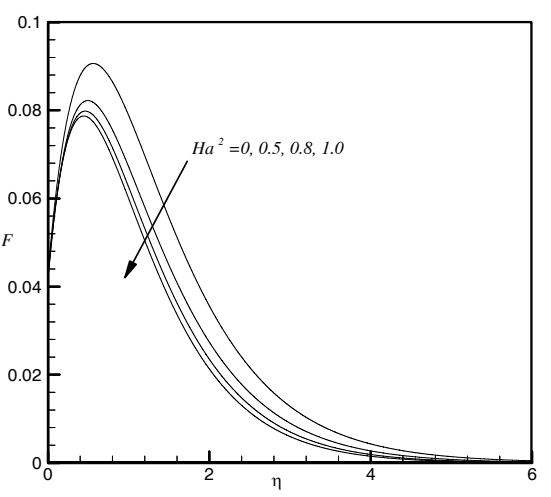

(a)

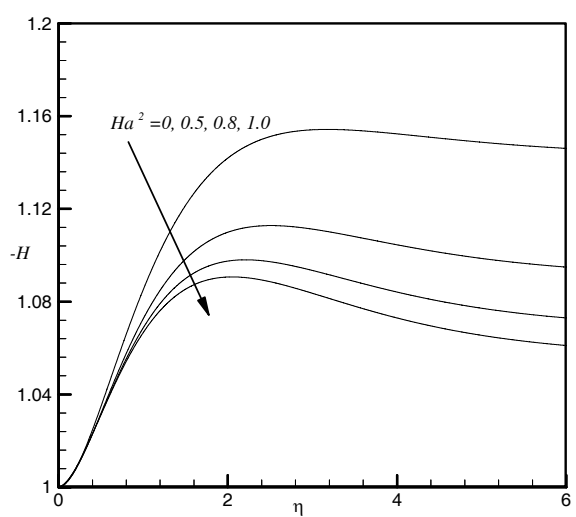

(c)

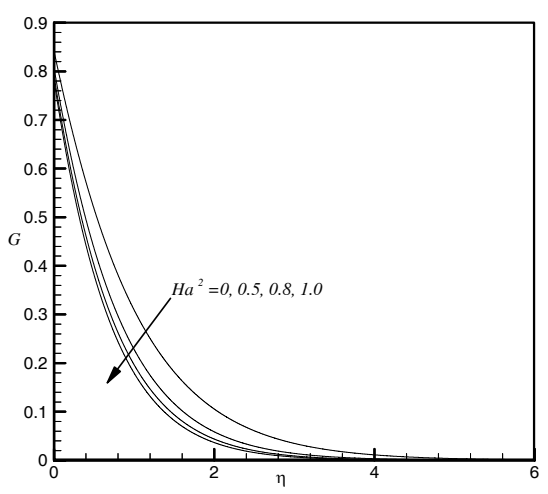

(b)

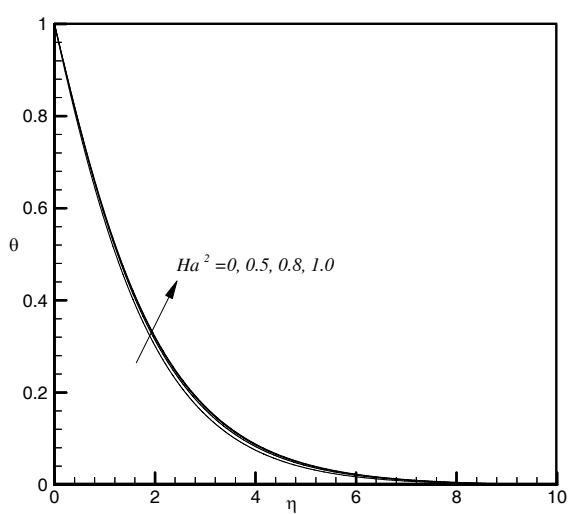

(d)

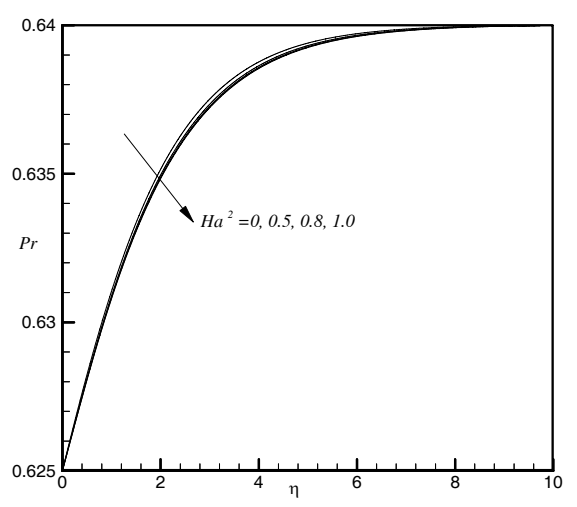

(e)

Figure 3. Variation of (a) radial velocity, (b) tangential velocity, (c) axial velocity, (d) temperature profile, and (e) variable Prandtl number for several values of $\mathrm{Ha}$. 


\section{CONVECTIVE HYDROMAGNETIC SLIP FLOW}

The effects of the suction (or injection) parameter $\left(w_{s}\right)$ on the radial, tangential and axial velocity profiles are shown in Figures 2 (a)-(c) respectively. From Figures 2(a)-(b) we see that radial and tangential velocity profiles decrease very rapidly as the suction velocity $\left(w_{s}<0\right)$ intensifies. The maximum of the radial velocity profiles moves towards the surface of the disk. It is also apparent that the thickness of the boundary layer decreases as suction velocity increases. Therefore, suction stabilizes the boundary layer growth. From Figure 2(c) we found that for strong suction, inward axial velocity is nearly constant. Figure 2(d) depicts the variation of the temperature profiles for various values of the suction parameter. The effect of the suction parameter on the thermal boundary layer is found to be similar to those of the radial and tangential velocity boundary layers. Applying suction, one can control the flow and heat transfer characteristics. In Figure 2(e) we have plotted variable Prandtl number as a function of $\eta$ to show the variation of the Prandtl number throughout the boundary for several values of the suction parameter. From this Figure we see that within the boundary layer for a fixed value of $\eta$ variable Prandtl number increases as the suction parameter increases while far away from the surface of the disk $\operatorname{Pr}$ equals its ambient value $\mathrm{Pr}_{\infty}$. An opposite effect is found for the case of fluid injection $\left(w_{s}>0\right)$.

The influence of the magnetic field parameter (Hartmann number) $H a$ on $F, G$, and $-H$ distributions is depicted in Figures 3(a)-(c). An increase in $\mathrm{Ha}$ induces a significant decrease in radial and tangential velocity profiles throughout the boundary layer; this is due to fact that imposition of a magnetic field to an electrically conducting fluid creates a drag force called the Lorentz force that has a tendency to slow down the flow around the disk at the expense of increasing its temperature. From Figure 3(c) it is also apparent that inward axial velocity decreases substantially with the increase of the Hartmann number. An increase in Hartmann number increases temperature profiles and hence increases the thermal boundary layer as can be seen from Figure 3(d). The variation of the Prandtl number within the boundary layer for different values of the Hartmann number is depicted in Figure 3(e). This Figure reveals that variable Prandtl number decreases with the increase of the Hartmann number.

In Figures 4(a)-(d), the influence of Hall current parameter $(m)$ on $F, G,-H$ and $\theta$ distributions across the boundary layer are given. The parameter $m$ has remarkable effect on the velocity profiles. It is observed that radial as well as inward axial velocity profiles increase as the Hall current parameter increases up to a certain value of $m<1$. Beyond this value of $m$, profiles of $F$ and $-H$ decrease with the further increase of $m$. It can be explained as follows: From equation (19) we see that the radial velocity term with Hall current is $-\frac{H a^{2}}{1+m^{2}} F(1+\gamma \theta)^{-a}$. An increase in $m(<1)$ will induce very minor alterations in the expression $\frac{1}{1+m^{2}}$. However in equation (20), the term $-\frac{H a^{2}}{1+m^{2}}(m F+G)(1+\gamma \theta)^{-a}$ gives an effective contribution to the radial velocity through $-\frac{H a^{2}}{1+m^{2}} m F(1+\gamma \theta)^{-a}$ indicating that an increase in $m(<1)$ causes a direct increase in the radial velocity. But for $m>1$ an opposite scenario is observed. Conversely we observe that the tangential velocity $(G)$ increases with an increase in Hall current parameter. From equation (20) we see that the tangential velocity is affected via the term $-\frac{H a^{2}}{1+m^{2}} G(1+\gamma \theta)^{-a}$, thus a change in $m$ produces very little effect, due to the inverse relationship of $m$ and the tangential velocity $G$. This effect will impede the tangential 


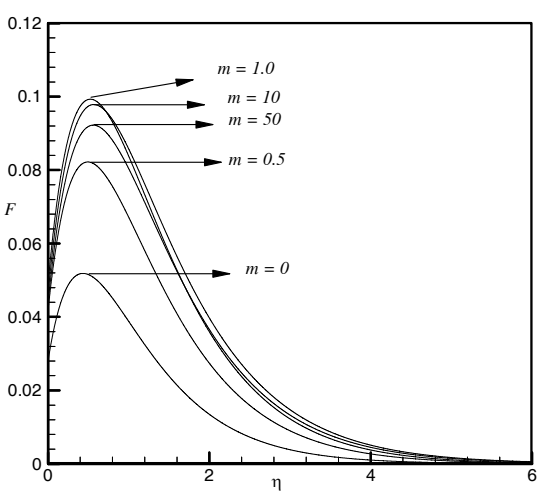

(a)

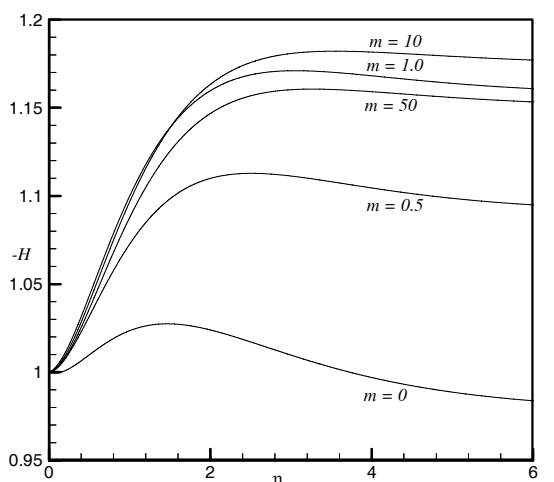

(c)

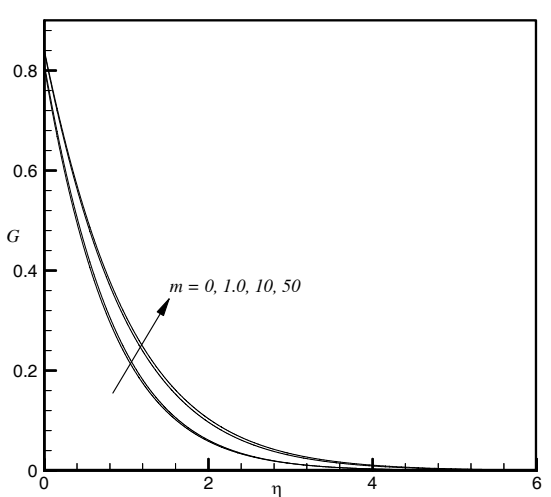

(b)

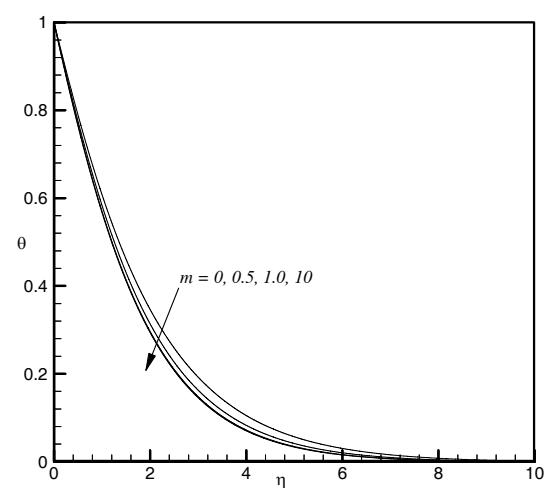

(d)

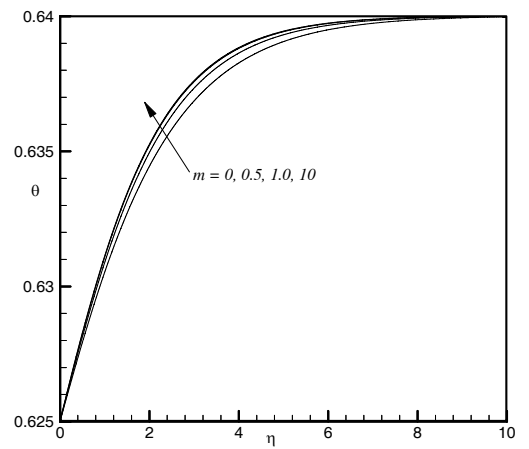

(e)

Figure 4. Variation of (a) radial velocity, (b) tangential velocity, (c) axial velocity, (d) temperature profile, and (e) variable Prandtl number for several values of $m$. 


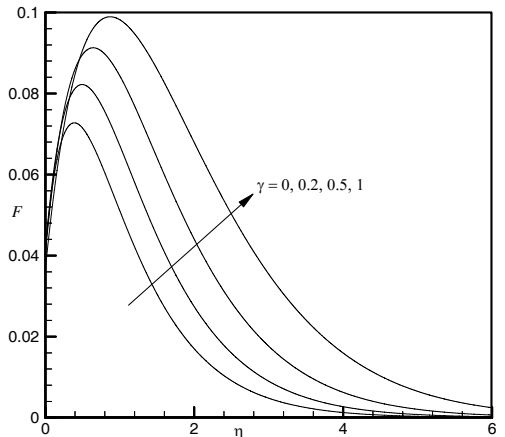

(a)

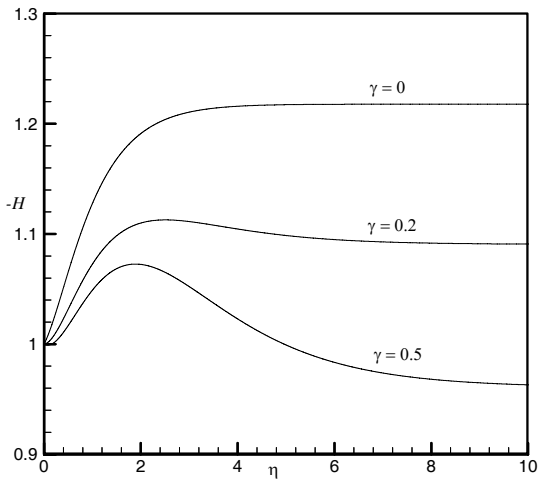

(c)

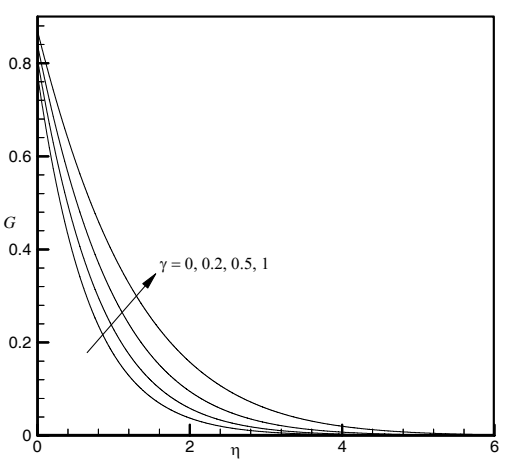

(b)

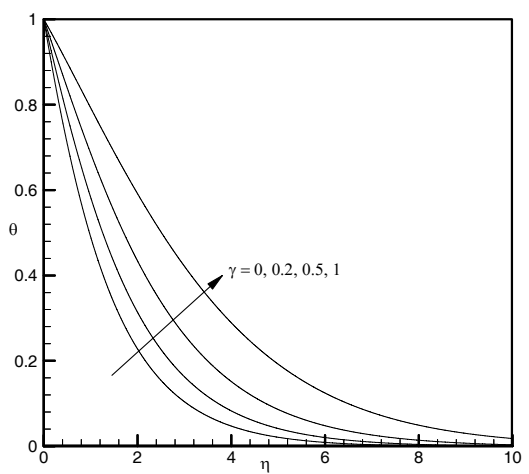

(d)

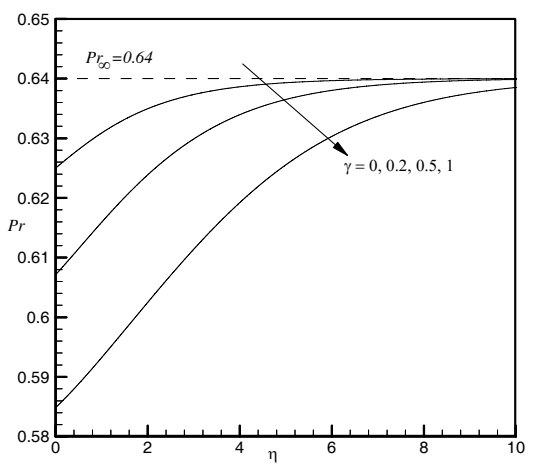

(e)

Figure 5. Variation of (a) radial velocity, (b) tangential velocity, (c) axial velocity, (d) temperature profile, and (e) variable Prandtl number for several values of $\gamma$. 
flow. However it will be swamped out by $\frac{H a^{2}}{1+m^{2}} m G(1+\gamma \theta)^{-a}$, the much greater direct proportionality in the factor affecting the tangential velocity in given equation (19). This positive term accelerates the flow for increase in $m$, explaining the rise in tangential velocity with increase in $m$. The mechanism by which Hall currents influence hydromagnetic disk flow is therefore via secondary effects and coupling in the momentum equations. Figure 4 (d) reveals that the thickness of the thermal boundary layer decreases as $m$ increases. The variation of the variable Prandtl number for different values of $m$ within the boundary layer is shown in Figure 4(e). It is clearly observed that an increase in $m$ increases Pr within the boundary layer. For very large values of $m$, increasing the effect of $m$ on $\operatorname{Pr}$ is less pronounced due to the fact that $\frac{1}{1+m^{2}}$ approaches to its limiting value 0 when $m \rightarrow \infty$, and as a consequence resistive effect of the magnetic field on the flow and temperature field is diminished.

Figures 5(a)-(d) explain the variation of the nondimensional radial, tangential, axial velocity and temperature profiles for various values of the relative temperature difference parameter $\gamma$. From Figure 5(a), we see that due to the existence of the centrifugal force the radial velocity increases and attains its maximum value for all values of $\gamma$. It is also observed that the maximum values of the radial velocity are 0.07276499 , $0.08221263,0.09128975$ and 0.09893225 for $\gamma=0,0.2,0.5$ and 1.0, respectively, and occur at $\eta=0.390$, $0.494,0.643$ and 0.871 , respectively. It is seen that the maximum velocity increases by $36 \%$ when $\gamma$ increases from 0 to 1.0. The case $\gamma=0$ corresponds to constant property of the working fluid. It is also seen that the smallest maximum value of the radial velocity is found for the case of constant property $(\gamma=0)$, which contradicts directly the findings of Maleque and Sattar (2005b). From Figure 5(b), it is found that the tangential velocity increases with the increasing values of $\gamma$. It can be seen from Figure 5(c) that inward axial velocity decreases with the increase of $\gamma$. It is also observed that close to the surface of the disk the effect of $\gamma$ gives rise to the familiar inflection point profile, which indicates that fluid with variable property on a highly heated surface, may lead to the destabilization of the laminar flow resulting in the development of the viscous sub-layer. Figure 5(d) depicts that temperature profile increases significantly with the increase of $\gamma$. Quantitatively, at $\eta=8.0$ the value of $\theta$ increases by $6026.8 \%$ when the value of $\gamma$ increases from 0 to 1.0. Thus the thickness of the thermal boundary layer increases markedly with the increase of $\gamma$ which is a direct contradiction to the findings of Maleque and Sattar (2005b), and Osalusi and Sibanda (2006). Studying a limited set of parameter values such as $\gamma=0,0.5$ (Maleque and Sattar, 2005b) and $\gamma=0,0.01$ (Osalusi and Sibanda, 2006) and considering Prandtl number as constant within the boundary layer, they concluded that an increase in $\gamma$ does not change the thickness of the thermal boundary layer. Figure 5(e) shows that variable Prandtl number $\operatorname{Pr}$ decreases very rapidly within the boundary layer for the increase of $\gamma$. For $\gamma=0$ variable Prandtl number $\operatorname{Pr}$ equals the ambient Prandtl number $\operatorname{Pr}_{\infty}$. For a fixed value of $\gamma(>0)$, $\operatorname{Pr}$ increases as $\eta$ increases and for $\eta \rightarrow \infty$, i.e. outside the boundary layer, it converges to its ambient value $\operatorname{Pr}_{\infty}$. From this figure it is also clear that at the surface of the disk (at $\eta=0$ ), $\gamma=0,0.2,0.5$, and 1 corresponds to $\operatorname{Pr}=0.64,0.625,0.607,0.585$ when other parameter values are fixed. Thus the effects of Pr on the velocity and temperature functions give the reverse effect of $\gamma$ on them. 


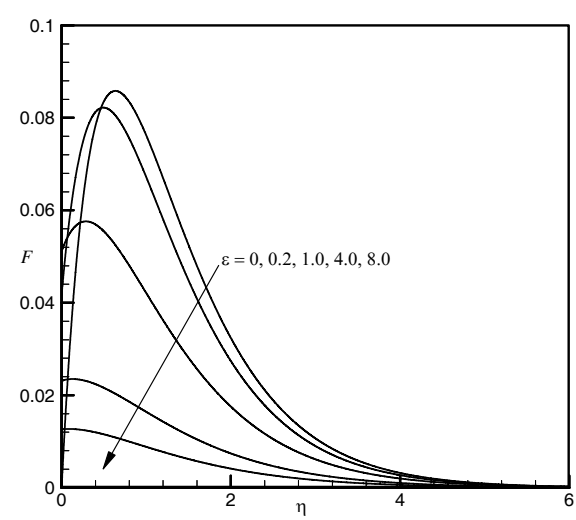

(a)

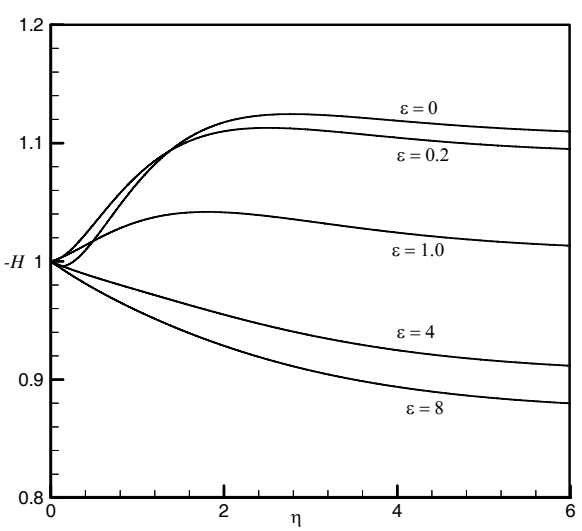

(c)

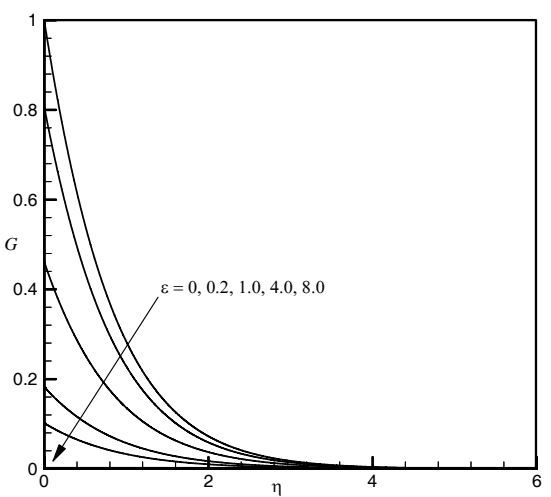

(b)

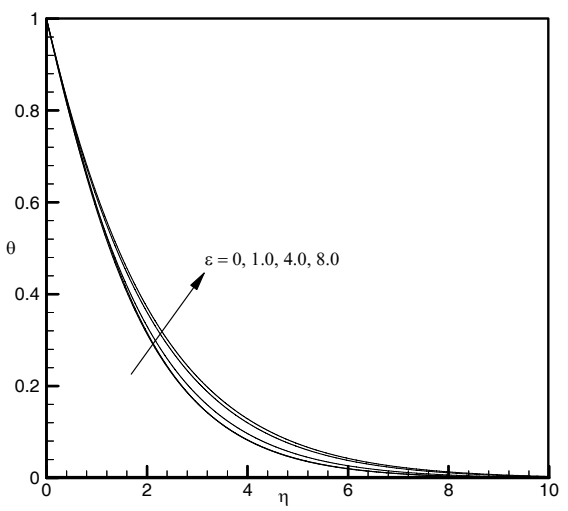

(d)

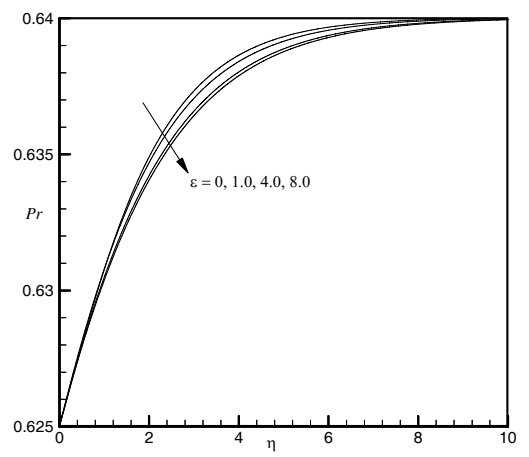

(e)

Figure 6. Variation of (a) radial velocity, (b) tangential velocity, (c) axial velocity, (d) temperature profile, and (e) variable Prandtl number for several values of $\varepsilon$. 


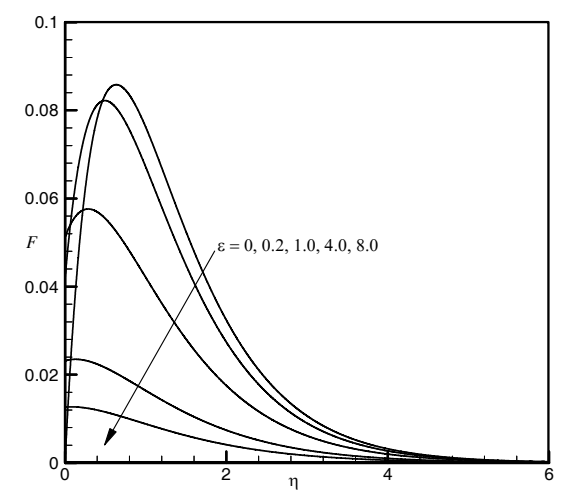

(a)

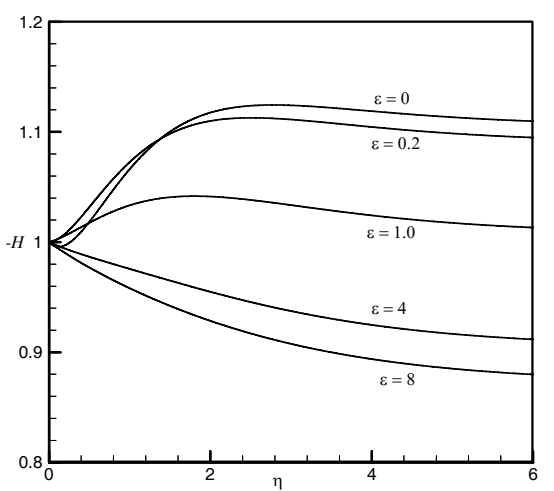

(c)

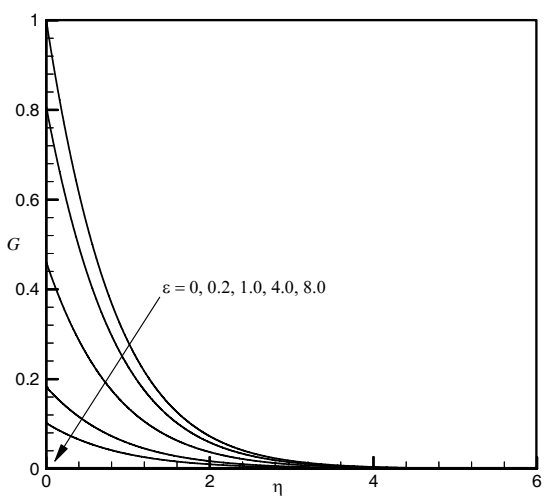

(b)

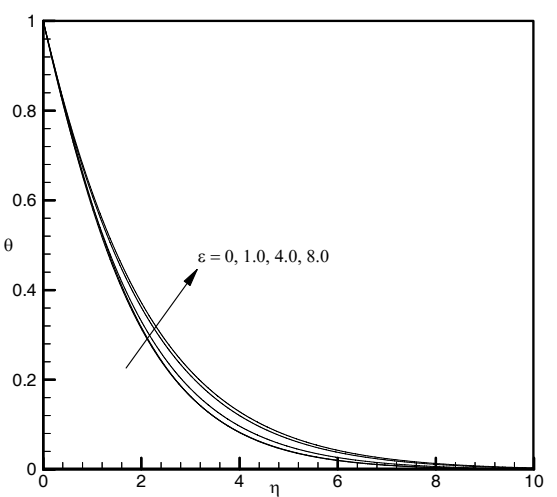

(d)

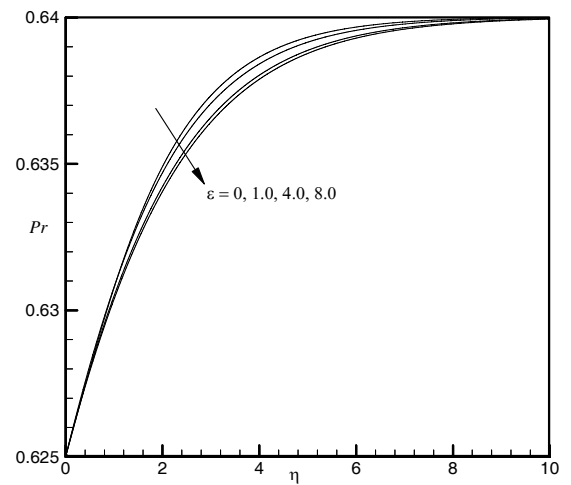

(e)

Figure 7. Variation of (a) radial velocity, (b) tangential velocity, (c) axial velocity, (d) temperature profile, and (e) variable Prandtl number for several values of $\varepsilon$. 


\section{CONVECTIVE HYDROMAGNETIC SLIP FLOW}

In Figures 6(a)-(d) we displayed velocity and temperature profiles for various values of the slip factor $(\varepsilon)$. Here $\varepsilon=0$ represents no-slip condition at the surface of the disk. From Figure 6(a) we see that the radial boundary layer decreases very rapidly with the increase of the slip factor. The thickness of the radial boundary layer is higher for no-slip flow compared to the slip flow. Fig. 6(a) further indicates that for large values of $\varepsilon$ i.e. $\varepsilon \rightarrow \infty$, the rotating disk does not cause rotation of the fluid particles. Because in this range of $\varepsilon$ the flow becomes entirely potential, there will be no motion in the fluid. This can be further explained as follows: the centrifugal force acting on the rotating disk (as like a centrifugal fan) will throw out the fluid that sticks to it. On the other hand, the flow in the axial direction will come forward to compensate for this thrown fluid. But increasing the slip on the surface of the disk reduces the amount of fluid that can stick on it; as a consequence the efficiency of the rotating disk is reduced substantially and is unable to transfer its circumferential momentum to the fluid particles. A reduction in the circumferential velocity results in a reduction in the centrifugal force which in turn decreases the inward axial velocity substantially as can be seen from Figure 6(c). From Figure 6(d) we see that the thermal boundary layer increases as slip factor $\varepsilon$ increases. Figure 6(e) shows a decreasing effect of $\varepsilon$ on the variable Prandtl number throughout the boundary layer.

In Table 5 we present skin-friction in radial and tangential directions and rate of heat transfer for various values of the pertinent parameters for a fixed value of $\mathrm{Pr}$. It can be seen that skin-friction in the radial direction decreases while skin-friction in the tangential direction increases with the increase of the suction parameter $w_{s}(<0)$. On the other hand, the rate of heat transfer increases with the increase of the suction parameter. An opposite effect is observed for the case of injection $\left(w_{s}>0\right)$.

Table 5 also shows that skin-friction in the radial direction increases for all increasing values of the Hartmann number except in the range of $0 \leq \mathrm{Ha}<0.707$ (not precisely determined). In this range of $\mathrm{Ha}$, radial skin-friction decreases as $\mathrm{Ha}$ increases. Tangential skin-friction increases while the rate of heat transfer decreases for all increasing values of the Hartmann number.

The effects of the Hall current parameter on the radial and tangential skin-frictions and the rate of heat transfer can be seen from Table 5. Skin-friction in the radial direction increases within the range of $0 \leq m \leq 1$. Outside of this range of $m$ an opposite behavior is observed. Tangential skin-friction decreases when $m$ increases within the range of $0 \leq m \leq 1$, and outside this range of $m$ tangential skin-friction increases with the further increase of $m$. The rate of heat transfer increases with the increase of $m$ for some $m<m_{c}$. But for the existence of strong Hall current i.e. $m>m_{c}$ the rate of heat transfer decreases with the further increase of $m$.

The effect of increasing Eckert number $E c$ has a decreasing effect on the radial skin-friction and on the rate of heat transfer whereas it has a very minor increasing effect on the tangential skin-friction as can be seen from Table 5 .

The variation of the radial and tangential skin-frictions and the rate of heat transfer for some selected values of the slip factor $\varepsilon$ are shown in Table 5. From here we see that skin-friction in both directions decreases with the increase of the slip factor. The largest skin-friction is found for the case of no-slip at the surface. On the other hand the rate of heat transfer increases with the increase of slip factor within the range of $0 \leq \varepsilon \leq 1$. But outside of this range of $\varepsilon$, the rate of heat transfer decreases with the further increase of the slip factor. Thus the rate of heat transfer can be strongly controlled by controlling the slip on the disk. 
Table 5. Numerical values of $F^{\prime}(0),-G^{\prime}(0)$ and $-\theta^{\prime}(0)$ for various values of $w_{s}, H a^{2}, m, E c$, and $\varepsilon$ with $\gamma=0.2, \operatorname{Pr}=0.625, a=0.7, b=0.83, d=-1.0$.

\begin{tabular}{|c|c|c|c|c|c|c|c|}
\hline$w_{s}$ & $H a^{2}$ & $m$ & $E c$ & $\varepsilon$ & $F^{\prime}(0)$ & $-G^{\prime}(0)$ & $-\theta^{\prime}(0)$ \\
\hline 0.5 & 0.5 & 0.5 & 0.2 & 0.2 & 0.30626574 & 0.57918115 & 0.05772346 \\
\hline 0.0 & 0.5 & 0.5 & 0.2 & 0.2 & 0.28341282 & 0.69042730 & 0.16076663 \\
\hline-0.5 & 0.5 & 0.5 & 0.2 & 0.2 & 0.24952322 & 0.81807709 & 0.30083407 \\
\hline-1.0 & 0.5 & 0.5 & 0.2 & 0.2 & 0.20853272 & 0.96222468 & 0.47057060 \\
\hline-2.0 & 0.5 & 0.5 & 0.2 & 0.2 & 0.12995186 & 1.28487051 & 0.86659713 \\
\hline-1.0 & 0.0 & 0.5 & 0.2 & 0.2 & 0.21477746 & 0.78866948 & 0.49905518 \\
\hline-1.0 & 0.5 & 0.5 & 0.2 & 0.2 & 0.20853272 & 0.96222468 & 0.47057060 \\
\hline-1.0 & 0.8 & 0.5 & 0.2 & 0.2 & 0.20907598 & 1.04165765 & 0.45885733 \\
\hline-1.0 & 1.0 & 0.5 & 0.2 & 0.2 & 0.21011307 & 1.08825472 & 0.45234655 \\
\hline-1.0 & 0.5 & 0.0 & 0.2 & 0.2 & 0.13956413 & 0.95761163 & 0.43868718 \\
\hline-1.0 & 0.5 & 0.5 & 0.2 & 0.2 & 0.20853272 & 0.96222468 & 0.47057060 \\
\hline-1.0 & 0.5 & 1.0 & 0.2 & 0.2 & 0.24406352 & 0.93179389 & 0.49107437 \\
\hline-1.0 & 0.5 & 10 & 0.2 & 0.2 & 0.23051412 & 0.80677029 & 0.50468008 \\
\hline-1.0 & 0.5 & 50 & 0.2 & 0.2 & 0.21822032 & 0.79212583 & 0.50044457 \\
\hline-1.0 & 0.5 & 0.5 & 0.0 & 0.2 & 0.20858805 & 0.96208379 & 0.53127404 \\
\hline-1.0 & 0.5 & 0.5 & 0.2 & 0.2 & 0.20853272 & 0.96222468 & 0.47057060 \\
\hline-1.0 & 0.5 & 0.5 & 0.4 & 0.2 & 0.20847731 & 0.96236597 & 0.40974692 \\
\hline-1.0 & 0.5 & 0.5 & 0.8 & 0.2 & 0.20836700 & 0.96264871 & 0.28795509 \\
\hline-1.0 & 0.5 & 0.5 & 1.0 & 0.2 & 0.20831208 & 0.96279014 & 0.22698731 \\
\hline-1.0 & 0.5 & 0.5 & 0.2 & 0.0 & 0.36777423 & 1.17657272 & 0.43706560 \\
\hline-1.0 & 0.5 & 0.5 & 0.2 & 0.2 & 0.20853272 & 0.96222468 & 0.47057060 \\
\hline-1.0 & 0.5 & 0.5 & 0.2 & 1.0 & 0.05117315 & 0.53881089 & 0.48991829 \\
\hline-1.0 & 0.5 & 0.5 & 0.2 & 4.0 & 0.00578668 & 0.20435375 & 0.47419596 \\
\hline-1.0 & 0.5 & 0.5 & 0.2 & 8.0 & 0.00158190 & 0.11227042 & 0.46583227 \\
\hline
\end{tabular}

Finally, the significance of the relative temperature difference $(\gamma)$ on the rate of heat transfer for both variable Prandtl number ( $V$ Pr ) and constant Prandtl number $(C \operatorname{Pr})$ is tabulated in Table 6. From this table we see that in both cases the rate of heat transfer from the surface of the disk to the fluid decreases for all increasing values of $\gamma$. We also see that rate of heat transfer for the variable property case is lower than the constant property case and the relative error between them increases significantly with the increase of $\gamma$. Therefore, consideration of Prandtl number as constant within the boundary layer for variable property is unrealistic. It is also mentionable that for our studied parameter values the relationship between the relative temperature difference parameter and the variable Prandtl number is an inverse relationship. So, the effect of $\operatorname{Pr}$ on the radial and tangential skin-frictions and on the rate of heat transfer is just the reverse of the effect of $\gamma$ on them. 
Table 6. Numerical values of $-\theta^{\prime}(0)$ for various values of $\gamma$ for $w_{s}=-1.0, \mathrm{Ha}^{2}=0.5, \mathrm{~m}=0.5$, $E c=0.2$, and $\varepsilon=0.2$ with $a=0.7, b=0.83, d=-1.0$.

\begin{tabular}{|c|c|c|c|}
\hline \multirow{2}{*}{$\gamma$} & \multicolumn{2}{|c|}{$-\theta^{\prime}(0)$} & \multirow{2}{*}{ Error $=\left|\frac{V \operatorname{Pr}-C \operatorname{Pr}}{V \operatorname{Pr}}\right| \times 100$} \\
\cline { 2 - 3 } & $V \operatorname{Pr}$ & $C$ Pr & $0.0 \%$ \\
\hline 0.0 & 0.64904702 & 0.64904702 & $1.2 \%$ \\
\hline 0.2 & 0.47057060 & 0.47627008 & $2.7 \%$ \\
\hline 0.5 & 0.31906666 & 0.32769165 & $4.6 \%$ \\
\hline 1.0 & 0.19338897 & 0.20237407 & $12.0 \%$ \\
\hline 3.0 & 0.05065792 & 0.05674936 & $24.3 \%$ \\
\hline 5.0 & 0.01661496 & 0.02066049 & \multicolumn{2}{|c}{} \\
\hline
\end{tabular}

\section{Conclusions}

In this study we experiment numerically on the effects of Hall current, viscous dissipation and Joule heating on hydromagnetic slip flow over a porous rotating disk taking into account the variable properties of the fluid. We illustrate the flow and heat transfer characteristics in terms of non-dimensional velocity and temperature profiles and tabulate skin-friction and rate of heat transfer, and show how the flow fields are influenced by the material parameters entering into the problem. As a result of computations the following conclusions can be drawn:

1. Suction stabilizes the boundary layer's growth.

2. Slip factor significantly controls the flow and heat transfer characteristics.

3. Increasing slip factor forces decrease of the Prandtl number within the boundary layer.

4. Hall parameter markedly controls the radial and axial flows. For strong Hall current (large $m>1$ ) flow along these directions decreases.

5. Hall current strongly controls the rate of heat transfer from the disk to the fluid. Very strong Hall current may reduce the heat transfer rate.

6. Hall current increases variable Prandtl number within the boundary layer.

7. The resistive effect of an applied magnetic field (Lorentz force) on the velocity and temperature profiles is apparent.

8. Increasing viscous dissipation parameter (or Eckert number) decreases the rate of heat transfer from the disk to the fluid.

9. The rate of heat transfer in a fluid of constant property is higher than - in a fluid of variable property.

10. The thickness of the thermal boundary layer is lower for a fluid of constant property than for a corresponding fluid of variable property.

11. For modeling thermal boundary layers with temperature dependent viscosity, Prandtl number must be treated as variable inside the boundary layer. 


\section{References}

ABOUL-HASSAN, A.L. and ATTIA, H.A. 1997. Flow due to a rotating disk with Hall effect. Phys. Lett. A., 228: 286-290.

ANDERSSON, H.I. and de KORTE, E. (2002). MHD flow of a power law fluid over a rotating disk. European J. Mech. B./Fluids, 21: 317-324.

ARIKOGLU, A. and OZKOL, I. 2006. On the MHD and slip flow over a rotating disk with heat transfer. Int. J. Numer Methods Heat Fluid Flow, 28: 172-184.

ATTIA, H.A. 1998. Unsteady MHD flow near a rotating porous disk with uniform suction or injection. Fluid Dyn. Res., 23: 283-290.

ATTIA, H.A. 2006. Unsteady flow and heat transfer of viscous incompressible fluid with temperature-dependent viscosity due to a rotating disc in a porous medium. J. Phys. A, Math. Gen., 39: 979-991.

BENTON, E.R. 1965. On the flow due to a rotating disc. J. Fluid Mech., 24: 781-800.

COCHRAN, W.G. 1934. The flow due to a rotating disc. Proc. Cambridge Phil. Soc. 30: 365-375.

EL-MISTIKAWY, T.M.A., ATTIA, H.A. and MEGAHED, A.A. 1991. The rotating disk flow in the presence of weak magnetic field. Proc. Fourth Conf. Theoret. Appl. Mech., Cairo, Egypt, 5-7 November 1991, pp. 6982.

EL-MISTIKAWY, T.M.A. and ATTIA, H.A. 1990. The rotating disk flow in the presence of strong magnetic field. Proc. Third int. Cong. Fluid Mech., Cairo, Egypt, 2-4 January 1990, 3: 1211-1222.

GAD-EL-HAK, M. 1999. The fluid mechanics of micro devices-the free scholar lecture. J. Fluid Eng-T. Asme., 121: 5-33.

HERRERO, J., HUMPHREY, J.A.C. and GIRALT, F. 1994. Comparative analysis of coupled flow and heat transfer between co-rotating discs in rotating and fixed cylindrical enclosures, Am. Soc. Mech. Eng., Heat Transfer Div. 300: 111-121.

HERWIG, H. and KLEMP, K. 1988. Variable property effects of fully developed laminar flow in concentric annuli. ASME J. Heat Trans., 110: 314-320.

JAYARAJ, S. 1995. Thermophoresis in laminar flow over cold inclined plates with variable properties. Heat Mass Trans., 40: 167-174.

KELSON, N. and DESSEAUX, A. 2000. Note on porous rotating disk flow. ANZIAM J. 42(E): C837-C855.

KUIKEN, H.K. 1971. The effect of normal blowing on the flow near a rotating disk of infinite extent. J. Fluid Mech., 47: 789-798.

MALEQUE, A.K. and SATTAR, A.M. 2005a. Steady laminar convective flow with variable properties due to a porous rotating disk. J. Heat Trans., 127: 1406-1409.

MALEQUE, A.K. and SATTAR, A.M. 2005b. The effects of variable properties and Hall current on steady MHD laminar convective fluid flow due to a porous rotating disk. Int. J. Heat Mass Trans., 48: 4963-4972.

MIKLAVCIC, M. and WANG, C.Y. 2004. The flow due to a rough rotating disk. Z. Angew. Math. Phys., 55: 235-246.

NACHTSHEIM, P.R. and SWIGERT, P. 1965. Satisfaction of asymptotic boundary conditions in numerical solution of system of nonlinear of boundary layer type. NASA TN-D3004.

OSALUSI, E., SIDE, J. and HARRIS, R. 2008. Thermal-diffusion and diffusion-thermo effects on combined heat and mass transfer of a steady MHD convective and slip flow due to a rotating disk with viscous dissipation and Ohmic heating. Int. Commu. Heat Mass Trans., 35: 908-915.

OSALUSI, E. and SIBANDA, P. 2006. On v ariable laminar convective flow properties due to a porous rotating disk in a magnetic field. Rom. J. Phys., 51: 933-944.

OWEN, J.M. and ROGERS, R.H. 1989. Flow and heat transfer in rotating disc system, Rotor-Stator Systems, vol. 1, Research Studies Press, Taunton, UK and John Wiley, NY.

ROGER, M.G. and LANCE, G.N. 1960. The rotationally symmetric flow of a viscous fluid in presence of infinite rotating disc. J. Fluid Mech., 7: 617-631. 


\section{CONVECTIVE HYDROMAGNETIC SLIP FLOW}

SPARROW, E.M., BEAVERS, G.S. and HUNG, L.Y. 1971. Flow about a porous-surface rotating disk. Int. J. Heat Mas Tranfer., 14: 993-996.

TAKHAR, H.S., SINGH, A.K. and NATH, G. (2002). Unsteady MHD flow and heat transfer on a rotating disk in an ambient fluid. Int. J. Therm. Sci., 41: 147-155.

VON KARMAN, T. 1921. Uber laminare und turbulente reibung. ZAMM, 1: 233-255.

ZAKERULLAH, M. and ACKROYD, J.A.D. 1979. Laminar natural convection boundary layers on horizontal circular discs. J. Appl. Math. Phys., 30: 427- 435.

Received 22 June 2009

Accepted 14 February 2010 